\title{
SK channel activation is neuroprotective in conditions of enhanced ER-mitochondrial coupling
}

Birgit Honrath ${ }^{1,2}$, Inge E. Krabbendam², Carmen IJsebaart ${ }^{2}$, Valentina Pegoretti ${ }^{2}$, Nadia Bendridi ${ }^{3}$, Jennifer Rieusset ${ }^{3}$, Martina Schmidt ${ }^{2}$, Carsten Culmsee (1) ${ }^{1}$ and Amalia M. Dolga ${ }^{1,2}$

\begin{abstract}
Alterations in the strength and interface area of contact sites between the endoplasmic reticulum (ER) and mitochondria contribute to calcium $\left(\mathrm{Ca}^{2+}\right)$ dysregulation and neuronal cell death, and have been implicated in the pathology of several neurodegenerative diseases. Weakening this physical linkage may reduce $\mathrm{Ca}^{2+}$ uptake into mitochondria, while fortifying these organelle contact sites may promote mitochondrial $\mathrm{Ca}^{2+}$ overload and cell death. Small conductance $\mathrm{Ca}^{2+}$-activated $\mathrm{K}^{+}(\mathrm{SK})$ channels regulate mitochondrial respiration, and their activation attenuates mitochondrial damage in paradigms of oxidative stress. In the present study, we enhanced ER-mitochondrial coupling and investigated the impact of SK channels on survival of neuronal HT22 cells in conditions of oxidative stress. Using genetically encoded linkers, we show that mitochondrial respiration and the vulnerability of neuronal cells to oxidative stress was inversely linked to the strength of ER-mitochondrial contact points and the increase in mitochondrial $\mathrm{Ca}^{2+}$ uptake. Pharmacological activation of SK channels provided protection against glutamate-induced cell death and also in conditions of increased ER-mitochondrial coupling. Together, this study revealed that SK channel activation provided persistent neuroprotection in the paradigm of glutamate-induced oxytosis even in conditions where an increase in ER-mitochondrial coupling potentiated mitochondrial $\mathrm{Ca}^{2+}$ influx and impaired mitochondrial bioenergetics.
\end{abstract}

\section{Introduction}

Multiple lines of evidence indicate that the etiologies of neurodegenerative disorders, such as Alzheimer's disease $(\mathrm{AD})$ or Parkinson's disease (PD) are strongly associated with common features of neuronal damage such as dysregulation of calcium $\left(\mathrm{Ca}^{2+}\right)$ homeostasis and oxidative stress $^{1-5}$. Disrupted $\mathrm{Ca}^{2+}$ homeostasis can lead to

Correspondence: Birgit Honrath (birgithonrath@gmail.com) or

Amalia M. Dolga (a.m.dolga@rug.nl)

${ }^{1}$ Institute of Pharmacology and Clinical Pharmacy, University of Marburg, 35043 Marburg, Germany

${ }^{2}$ Faculty of Science and Engineering, Groningen Research Institute of Pharmacy (GRIP), Research School of Behavioural and Cognitive Neurosciences (BCN), Department of Molecular Pharmacology, University of Groningen, 9713 AV Groningen, The Netherlands

Full list of author information is available at the end of the article. These authors contributed equally: Birgit Honrath, Inge E. Krabbendam.

Edited by A. Verkhratsky mitochondrial $\mathrm{Ca}^{2+}\left(\left[\mathrm{Ca}^{2+}\right]_{\mathrm{m}}\right)$ overload, and subsequently to an impairment of mitochondrial energy metabolism and respiration ${ }^{6,7}$. Under physiological conditions however, $\left[\mathrm{Ca}^{2+}\right]_{\mathrm{m}}$ is a prerequisite for aerobic energy metabolism through the regulation of oxidative phosphorylation (OXPHOS) and mitochondrial ATP production ${ }^{8,9}$.

Close spatial interactions between the endoplasmic reticulum (ER) and mitochondria are essential for rapid and sustained $\left[\mathrm{Ca}^{2+}\right]_{\mathrm{m}}$ uptake. These close contacts are established at the so called mitochondria-associated ER membranes (MAM), thereby facilitating $\mathrm{Ca}^{2+}$ transfer between ER and mitochondria through mitochondrial voltage-dependent anion channels (VDAC) and ERlocated inositol-1,4,5-trisphosphate receptors $\left(\mathrm{IP}_{3} \mathrm{R}\right)$, which are physically connected by glucose-regulated

\section{(c) The Author(s) 2018}

(c) (i) Open Access This article is licensed under a Creative Commons Attribution 4.0 International License, which permits use, sharing, adaptation, distribution and reproduction in any medium or format, as long as you give appropriate credit to the original author(s) and the source, provide a link to the Creative Commons license, and indicate if changes were made. The images or other third party material in this article are included in the article's Creative Commons license, unless indicated otherwise in a credit line to the material. If material is not included in the article's Creative Commons license and your intended use is not permitted by statutory regulation or exceeds the permitted use, you will need to obtain permission directly from the copyright holder. To view a copy of this license, visit http://creativecommons.org/licenses/by/4.0/. 
protein 75 (GRP75 $^{10-12}$. Mutations in MAM-associated proteins have been identified to either enhance or reduce ER-mitochondrial coupling (EMC), thereby leading to dysregulation of MAM interfaces and progressive neuronal degeneration as shown in models of $\mathrm{AD}$ and amyotrophic lateral sclerosis ${ }^{13-15}$.

In neuronal cells, activation of small conductance $\mathrm{Ca}^{2+}$-activated $\mathrm{K}^{+}$(SK) channels regulated $\mathrm{Ca}^{2+}$ uptake and retention in the $\mathrm{ER}^{16}$, and also controlled mitochondrial $\mathrm{Ca}^{2+}$ homeostasis and respiration ${ }^{17}$. Activation of SK channels in conditions of ER stress and glutamateinduced oxidative stress (oxytosis) preserved cell viability, and restored ER and mitochondrial function, respectively. In this study, we aimed to investigate the ability of SK channels to confer protection following oxidative stress in conditions where EMC was increased.

\section{Results}

Inducing a physical linkage between ER and mitochondria

ER and mitochondria temporarily and dynamically form close contacts at the MAM, thereby allowing for the exchange of proteins, lipids, and ions between the organelles ${ }^{18}$. To study the structure and function of ER-mitochondrial connections, we used genetically encoded bifunctional linkers ${ }^{19}$ that both, tighten the contact between ER and mitochondria, and expand the MAM interface area. These linkers consist of OMMFKBP12-mRFP and ER-targeted-FRB-CFP fusion proteins which heterodimerize in response to rapamycin application at very low concentrations $(100 \mathrm{nM})$.

Upon application of rapamycin, we observed a timedependent co-localization of CFP-tagged ER with RFPtagged mitochondria indicating the induction of EMC, reaching complete co-localization after $10 \mathrm{~min}$ following rapamycin treatment (Fig. 1a). In contrast, a GFP-tagged ER-Flipper control plasmid (FL), co-transfected with OMM-FKBP12-mRFP, failed to co-localize with RFPtagged mitochondria following rapamycin treatment (Fig. 1b), which confirmed the specificity of the hereafter called ER-mitochondrial linkers (EML).

Enhancing EMC increases $\left[\mathrm{Ca}^{2+}\right]_{\mathrm{m}}$ uptake and impairs the mitochondrial reserve respiratory capacity

The rapamycin-induced heterodimerization of the genetically encoded linkers tightens ER-mitochondrial contact points and increases the surface of the MAM interface ${ }^{19}$, which might amplify $\mathrm{Ca}^{2+}$ transfer into the mitochondrial matrix and modulate mitochondrial respiration ${ }^{20}$.

To this end, we assessed $\left[\mathrm{Ca}^{2+}\right]_{\mathrm{m}}$ uptake in the presence of the linkers and their pharmacological inducer rapamycin. In order to study fast changes in $\left[\mathrm{Ca}^{2+}\right]_{\mathrm{m}}$ uptake induced by strengthening EMC, we utilized a system to perform realtime $\left[\mathrm{Ca}^{2+}\right]_{\mathrm{m}}$ uptake measurements in HEK293T cells transfected with the linkers and a mitochondrial GFPaequorin $^{21}$. Up to $80 \%$ of HEK293T cells on average were transfected with the wild type $\left(\mathrm{mtGA}^{\mathrm{wt}}\right)$ or a $\mathrm{Ca}^{2+}$-binding deficient mutant aequorin version ( $\mathrm{mGA}^{\mathrm{mut}}$ ) serving as an internal control (Fig. 1c). Calibration of the aequorin sensor revealed a dose-dependent increase in the luminescence with increasing $\mathrm{CaCl}_{2}$ concentrations (Figure S1a-b). Stimulation of HEK293T cells transfected with the linkers and co-expressing $\mathrm{mtGA}^{\mathrm{wt}}$ with $50 \mathrm{mM} \mathrm{CaCl}{ }_{2}$ induced $\left[\mathrm{Ca}^{2+}\right]_{\mathrm{m}}$ uptake as shown by an elevation of the luminescence signal (Fig. 1d) and of the total luminescence (Fig. 1e) compared to cells stimulated with control solution. Importantly, stimulation of linker-transfected HEK293T cells which were pre-treated with $100 \mathrm{nM}$ rapamycin during sensor reconstitution, resulted in higher $\left[\mathrm{Ca}^{2+}\right]_{\mathrm{m}}$ uptake compared to control and untreated cells, suggesting that EMC strengthening increased $\left[\mathrm{Ca}^{2+}\right]_{\mathrm{m}}$ uptake. In contrast, $\left[\mathrm{Ca}^{2}\right.$ $\left.{ }^{+}\right]_{\mathrm{m}}$ uptake in cells transfected with the $\mathrm{Ca}^{2+}$-binding deficient mutant ( $\mathrm{mtGA}^{\mathrm{mut}}$ ) was unchanged, indicating the specific binding of $\mathrm{Ca}^{2+}$ to the $\mathrm{tGA}^{\mathrm{wt}}$ construct (Fig. 1f). The treatment of transfected HEK293T cells with rapamycin did not affect cell viability as measured by the MTT Assay (Figure S1c).

Next, we determined the effects of strengthening EMC on mitochondrial bioenergetics in HT22 cells using Seahorse extracellular flux analyses. Mitochondrial respiration, assessed as the oxygen consumption rate (OCR), was recorded in real-time following the application of $200 \mathrm{nM}$ rapamycin over a period of $2 \mathrm{~h}$. In cells transfected with the Flipper control construct (Fig. 2, gray symbols/bars), rapamycin application did change the OCR compared to untreated control cells. In contrast, rapamycin application to linker-transfected (Fig. 2, blue symbols/bars) cells did not change basal respiration (Fig. 2b) but seemed to affect ATP production (Fig. 2c), as assessed following oligomycin treatment. Interestingly, upon uncoupling of the respiratory chain using dinitrophenol (DNP), the induced maximal OCR was decreased only in linker-transfected cells in the presence of rapamycin compared to untreated control cells (Fig. 2d) indicating an effect on the reserve respiratory capacity. Final inhibition of mitochondrial complexes I (rotenone) and III (antimycin A), therefore, resulted in a lower OCR in linker-transfected cells where rapamycin was applied (Fig. 2e). Notably, the rapamycininduced impairment of mitochondrial respiration in cells where EMC was increased, did not affect cell survival since cell proliferation was not impaired compared to Flipper-transfected control cells, as assessed using realtime impedance measurements (Figure S2a). Further, formation of the linker dimer did not affect ER integrity or function since the mRNA of $C H O P$, a classical marker for ER stress, was not changed following rapamycin treatment in either Flipper-transfected or linkertransfected cells (Figure S2b). 


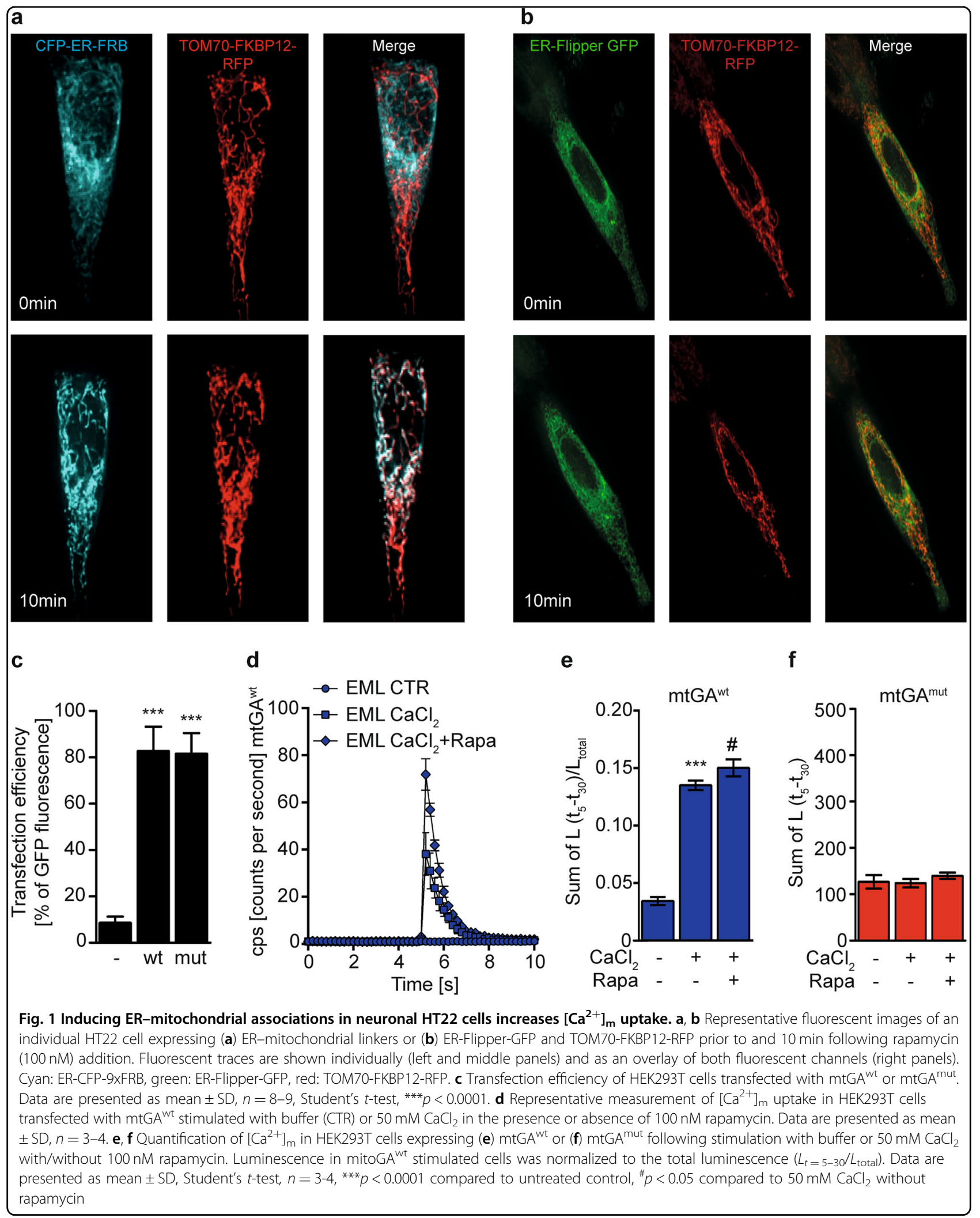


a Oxygen Consumption Rate
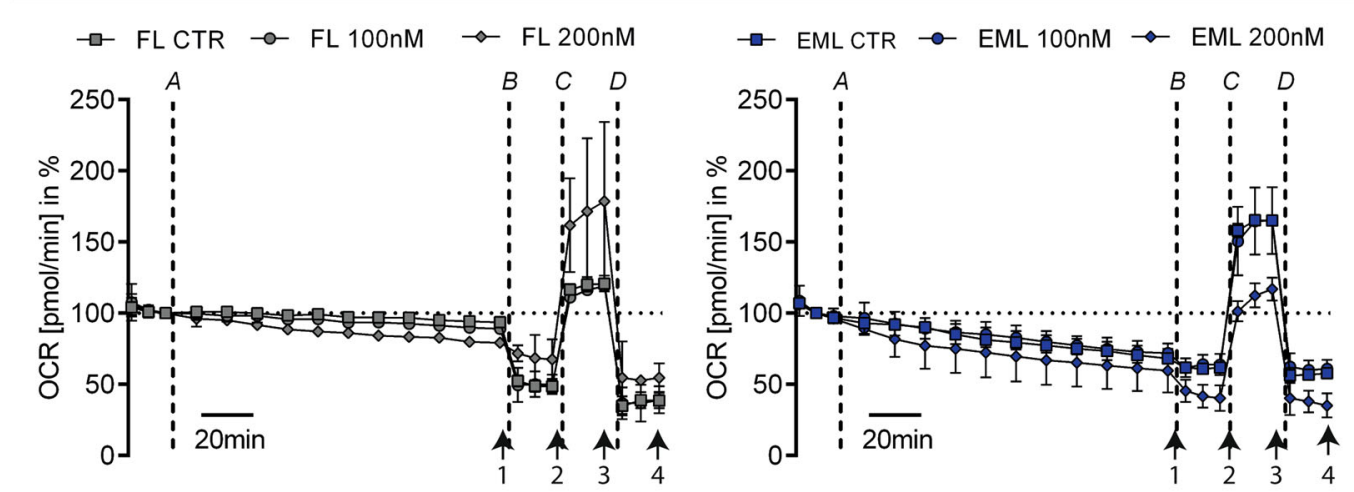

b

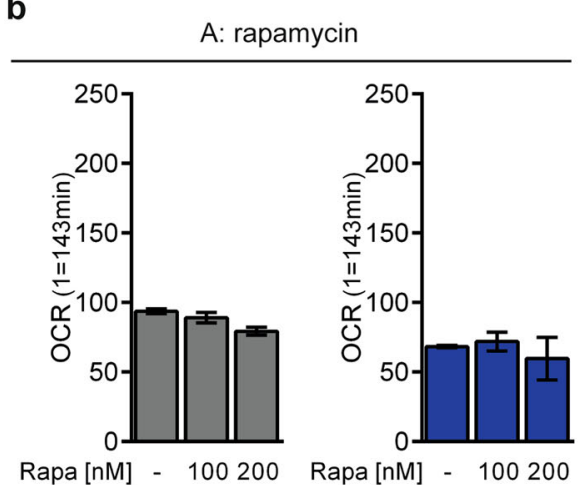

C

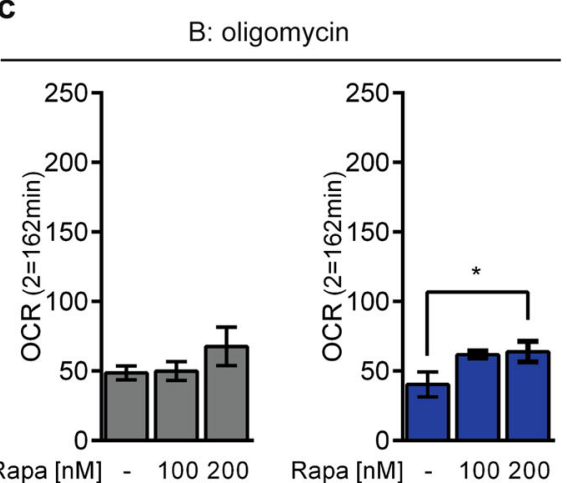

d

C: DNP
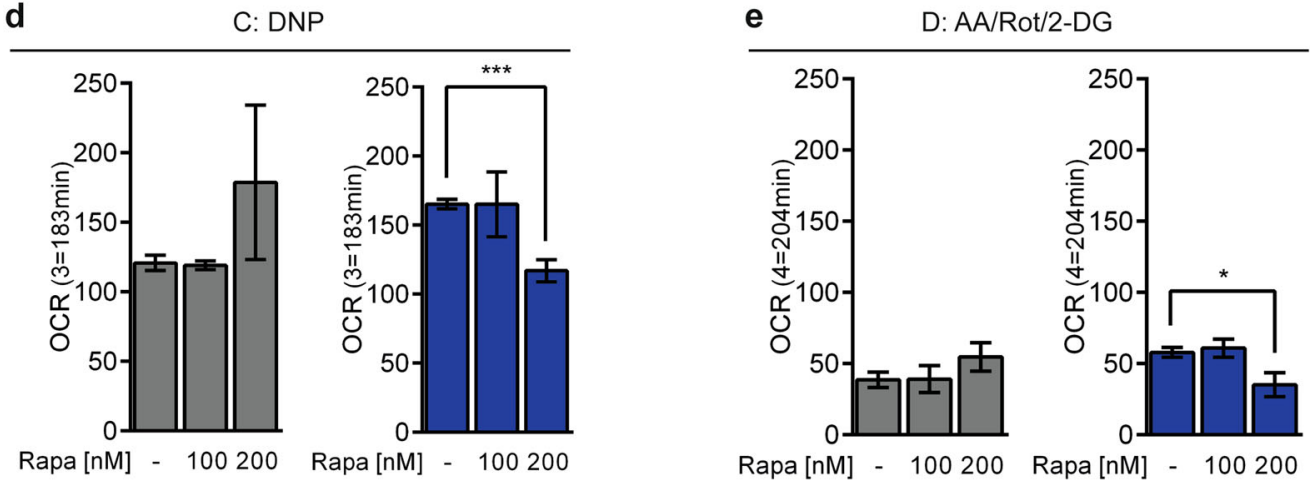

Fig. 2 Enhancing ER-mitochondrial coupling impairs mitochondrial respiration. a Representative measurement of the oxygen consumption rate (OCR) in HT22 cells transfected with TOM70-FKBP12-mRFP and Flipper control plasmid (named FL, gray symbols/bars) or together with CFP-FRBER (named EML, blue symbols/bars) following the application of $100 \mathrm{nM}$ rapamycin. A: rapamycin, B: oligomycin, C: DNP, D: antimycin A, rotenone, 2DG. Data are presented as mean $\pm S D, n=3-6$ per condition. Arrows indicate analyzed time points. $\mathbf{b}$-e Quantification of the OCR at the indicated time points following the application of $\mathbf{b}$ rapamycin, $\mathbf{c}$ oligomycin, $\mathbf{d}$ DNP, and $\mathbf{e}$ antimycin A, rotenone, 2-DG. Student's t-test, ${ }^{* * *} p<0.0001$ compared to untreated control

Taken together, we show that promoting EMC specifically amplified $\left[\mathrm{Ca}^{2+}\right]_{\mathrm{m}}$ influx, affected ATP production, and impaired the reserve respiratory capacity, without affecting basal respiration, cell viability, or proliferation.

\section{Strengthening EMC accelerates neuronal cell death}

To study the consequences of increased EMC on neuronal cell death signaling, we induced oxidative stress and mitochondrial damage in neuronal HT22 cells by glutamate $^{22}$.

In HT22 cells, cell death was detectable approximately 12 $\mathrm{h}$ following glutamate exposure with maximal damage detected after $16-18 \mathrm{~h}$, as indicated by the MTT assay (Fig. 3a). Since rapamycin can induce mammalian target of rapamycin (mTOR) signaling and thereby autophagy ${ }^{23}$, we controlled for cell viability and cell death induction in the 


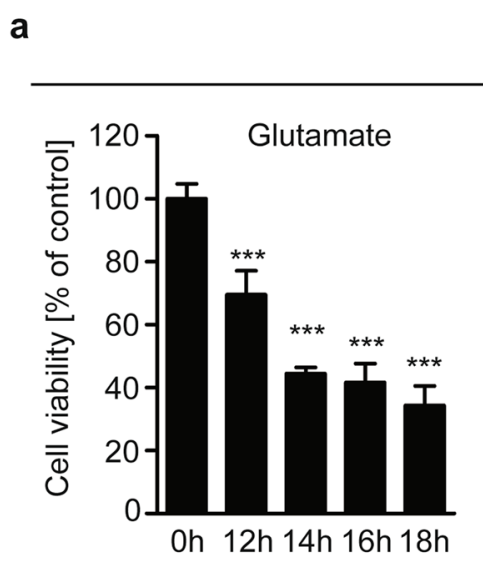

C

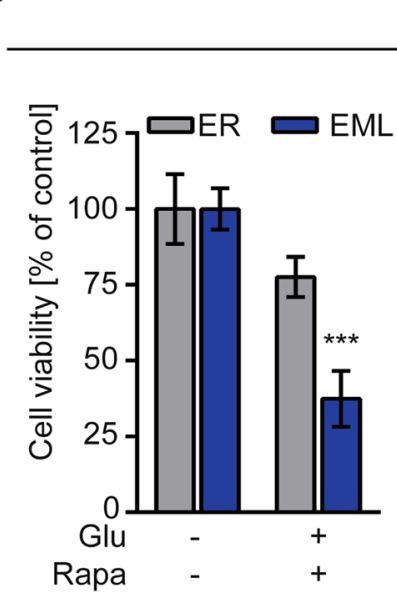

b

untransfected

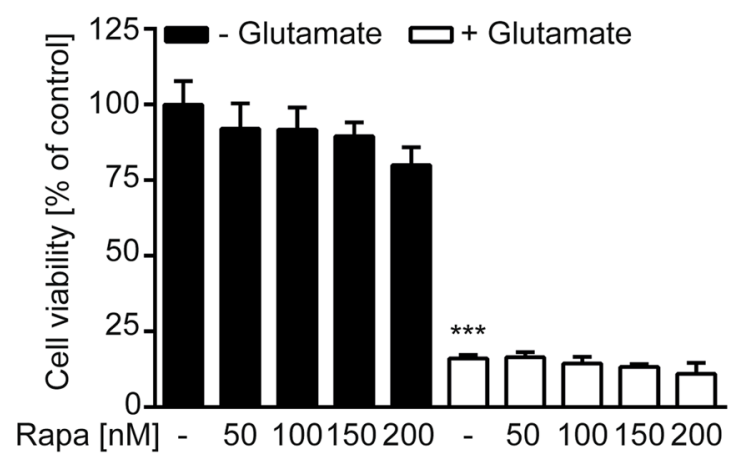

d

Flipper/Linker transfection

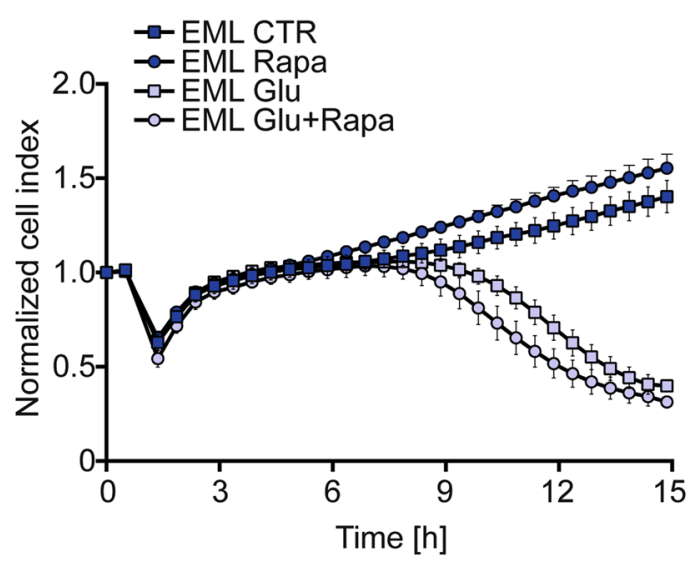

Fig. 3 Strengthening EMC potentiates neuronal cell death. a MTT Assay following glutamate treatment ( $8 \mathrm{mM}$ ) for $12-18 \mathrm{~h}$. Data are presented as mean $\pm S D, n=8$, Student's $t$-test ${ }^{* *} p<0.0001$ compared to untreated control. $\mathbf{b}$ MTT assay following treatment with glutamate $(8 \mathrm{mM}, 16 \mathrm{~h})$ in the presence or absence of rapamycin $(0,50,100,150,200 \mathrm{nM})$. Data are presented as mean $\pm S D, n=6$, Student's $t$-test ${ }^{* * *} p<0.0001$ compared to untreated control. $\mathbf{c}$ xCELLigence measurement of HT22 cells transfected with ER-FRB-CFP and TOM70-FKBP12-RFP (EML) following addition of glutamate $(8 \mathrm{mM}, 16 \mathrm{~h})$ in the presence or absence of rapamycin $(100 \mathrm{nM})$. Data are presented as mean $\pm S D, n=6$. $\mathbf{d}$ MTT Assay in HT22 cells transfected with TOM70-FKBP12-RFP and either ER-CFP (ER, gray bars) or ER-FRB-CFP (EML, blue bars) following treatment with glutamate (4 mM, 16 h) and rapamycin $(100 \mathrm{nM})$. Data are presented as mean $\pm S D, n=6$, Student's $t$-test ${ }^{* *} p<0.0001$ compared to glutamate in ER control

presence of rapamycin in neuronal HT22 cells. Rapamycin alone neither affected cell viability under control conditions nor glutamate toxicity (Fig. 3b), indicating that rapamycin at the low concentrations used in our study did not induce mTOR-dependent signaling nor autophagy.

Next, we investigated the consequences of rapamycinmediated EMC, via linker heterodimerization, on cell viability using the MTT assay. Glutamate reduced cell viability in both, ER-transfected control and linker-transfected cells, yet cell death was pronounced in linker-transfected cells following rapamycin treatment (Fig. 3c, blue bars). We confirmed these pronounced effects on proliferation using real-time cellular impedance measurements showing that the addition of rapamycin to linker-transfected cells accelerated cell death induction by glutamate, represented as a drop in the cellular impedance curve (Fig. 3d). These results suggest that enhanced EMC accelerated glutamateinduced neuronal cell death.

\section{Activation of small conductance $\mathrm{Ca}^{2+}$-activated $\mathrm{K}^{+}$(SK) channels preserves cell survival in conditions of enhanced EMC}

Small conductance $\mathrm{Ca}^{2+}$-activated $\mathrm{K}^{+}$(SK) channels have been identified in the ER membrane ${ }^{16}$, as well as in the inner mitochondrial membrane (IMM) ${ }^{17}$ of neuronal cells where they contribute to cell survival in conditions of stress. Their activation with the SK $2 / 3$ channel activator $\mathrm{CyPPA}^{24}$ reduced mitochondrial respiration, which prevented glutamate-induced mitochondrial dysfunction and cell death ${ }^{17}$. 
Here, we assessed the effects of SK channel activation in conditions of strengthened EMC on neuronal toxicity induced by glutamate. Therefore, we treated linkertransfected HT22 cells with rapamycin and challenged them with glutamate in the presence or absence of CyPPA. The treatment with CyPPA alone did not alter cell survival in cells transfected either with the Flipper control plasmid or the linkers, as assessed by cellular impedance measurements (Figure S3). In agreement with our previous study ${ }^{17}$, CyPPA protected against cell death induced by oxytosis. Here, we now show that neuroprotection was still present even in conditions of strengthened EMC (Fig. 4a). We also challenged linker-transfected HT22 cells that were co-transfected with either wild-type SK2 channels (SK2) or mitochondria-targeted SK2 channels (mitoSK2), with glutamate in the presence of rapamycin to induce linkage formation. Overexpression of either SK2 or mitoSK2 (Fig. 4b) combined with CyPPA preserved cell viability following oxytosis, and cell survival was enhanced in cells transfected with SK2/mitoSK2 compared to the GFP/mitoGFP control plasmids. These results indicate that the overexpression of SK2 channels increased CyPPA-mediated protection against glutamate toxicity. Further, we investigated if the enhanced neuroprotective action of SK2 channel activation and/or overexpression was based on effects on the physical EMC. Using a proximity ligation approach, we assessed $I_{3} R 1-V D A C 1$ interactions and found that neither CyPPA treatment (Fig. 4d) nor the overexpression of wild-type or mitochondrial SK2 channels (Fig. 4e) influenced $\mathrm{IP}_{3} \mathrm{R} 1-\mathrm{VDAC} 1$ interaction sites.

These findings show that the pharmacological activation of SK channels still provides protection even in conditions of enhanced EMC, indicating that CyPPA could overcome the additional damage through enhanced $\left[\mathrm{Ca}^{2+}\right]_{\mathrm{m}}$ and reduced mitochondrial respiration. Increasing the expression of both, wild-type and mitoSK2 channels provides an additive effect on CyPPA-mediated protection. Interestingly, neuroprotective SK2 channel actions were independent of the physical EMC.

\section{Discussion}

Recent advances in the knowledge on the pathology of neurodegenerative diseases revealed a strong impact of EMC, MAM formation, and associated $\left[\mathrm{Ca}^{2+}\right]_{\mathrm{m}}$ transfer on mitochondrial metabolism and neuronal cell death $^{25-27}$. Weakening the ER-mitochondrial interface has therefore emerged as a strategy to prevent the development of neurodegenerative diseases ${ }^{28,29}$. In the present study, we provide evidence that increasing EMC in neuronal HT22 cells potentiated cell death in a model of oxytosis. We further investigated the impact of SK channels on cell viability in these conditions, since pharmacological SK channel activation provided neuroprotection in different disease paradigms in vitro and in vivo by regulating $\mathrm{Ca}^{2+}$ homeostasis.

ER and mitochondria are physically linked mainly by a trimeric complex consisting of ER-bound $\mathrm{IP}_{3} \mathrm{R}, \mathrm{VDAC}$ on the OMM and the molecular chaperone GRP7 $5^{11,30,31}$. Recently, it has been shown that ER-mitochondrial contact points can be formed using drug-inducible fluorescent organelle linkers at the ER membrane and outer mitochondrial membrane, which bring ER and mitochondria into close proximity $(<10 \mathrm{nM})^{19}$. In the current study, we expressed these drug-inducible EML in immortalized hippocampal HT22 cells, and confirmed the rearrangement of ER and mitochondria upon rapamycin treatment using live-cell imaging. Similar to Csordás and colleagues $^{19}$, we observed full co-localization of the CFPtagged ER plasmid and RFP-tagged mitochondrial plasmids, indicating a pronounced increase in EMC within minutes following addition of rapamycin.

ER-mitochondrial $\mathrm{Ca}^{2+}$ transfer and $\left[\mathrm{Ca}^{2+}\right]_{\mathrm{m}}$ load are critical factors to maintain mitochondrial function, with alterations in these processes promoting mitochondrial dysfunction. For instance, enhanced EMC provoked by the saturated fatty acid palmitate disrupted intracellular $\mathrm{Ca}^{2+}$ homeostasis, thereby promoting hepatotoxicity ${ }^{32}$, and enhanced VDAC-mediated $\left[\mathrm{Ca}^{2+}\right]_{\mathrm{m}}$ influx amplified cell death induction in HeLa cells ${ }^{10,33}$. Here, we utilized mitochondria-targeted GFP-aequorin ${ }^{21}$ measurements to confirm that strengthening EMC by inducing heterodimerization of the linkers in HEK293T cells specifically increased $\mathrm{Ca}^{2+}$ uptake into the mitochondria. $\mathrm{Ca}^{2+}$ signaling through the MAM interface also determines the cellular bioenergetic state, as shown for $\mathrm{Ca}^{2+}$ release through $\mathrm{IP}_{3} \mathrm{R}^{20,34,35}$ and the interaction of mitofusin 1 and mitofusin $2^{36}$. We observed that the increase in $\left[\mathrm{Ca}^{2+}\right]_{\mathrm{m}}$ influx, elicited by heterodimerization of the EML, was accompanied by a decrease in ATP production and in the mitochondrial reserve respiratory capacity. In contrast, in a model of ER stress, EMC was increased at early stages following ER stress induction, which was accompanied by an initial increase in mitochondrial respiration before it was attenuated concomitantly with the onset of cell death $(20 \mathrm{~h})^{34}$. The reason for these different effects may be that EMC induced by the bifunctional linkers was a rather fast and highly efficient process occurring within minutes compared to the ER stress-induced increase in EMC (1-4h) observed by Bravo and colleagues ${ }^{34}$. Thus, we propose that basal mitochondrial respiration was stable without an initial increase due to the speed and strength of the artificial linkage induced by the linkers.

The intensity of ER-mitochondrial associations therefore also regulates local $\mathrm{Ca}^{2+}$ homeostasis, and stressinduced $\mathrm{Ca}^{2+}$ dysregulation in both organelles is detrimental ${ }^{37,38}$. To this end, we investigated cell survival following stress induction in HT22 cells expressing the 
a

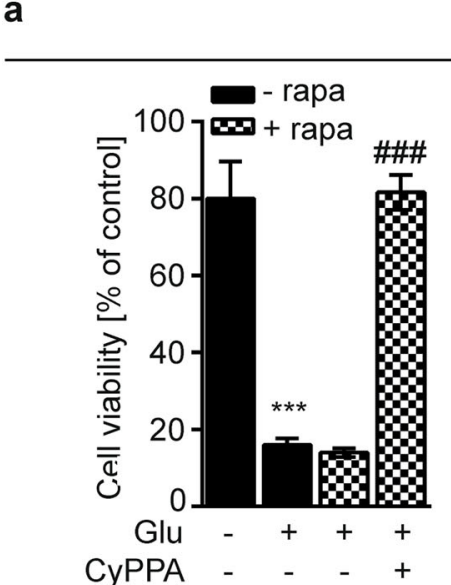

b d untransfected
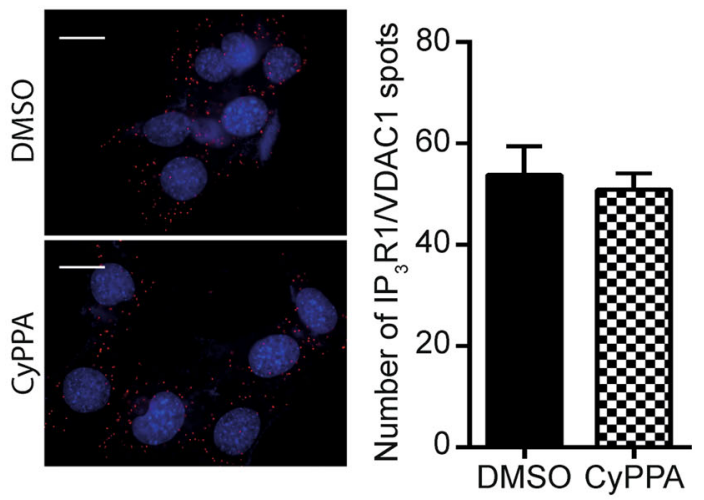

e

(mito)SK2 channel overexpression
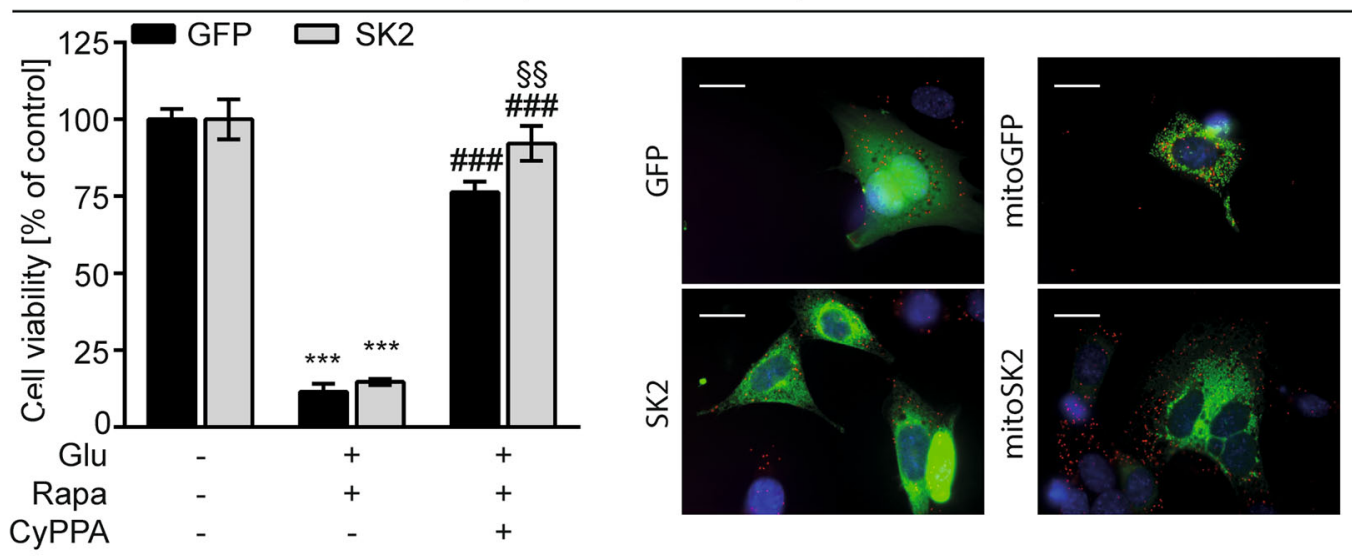

C
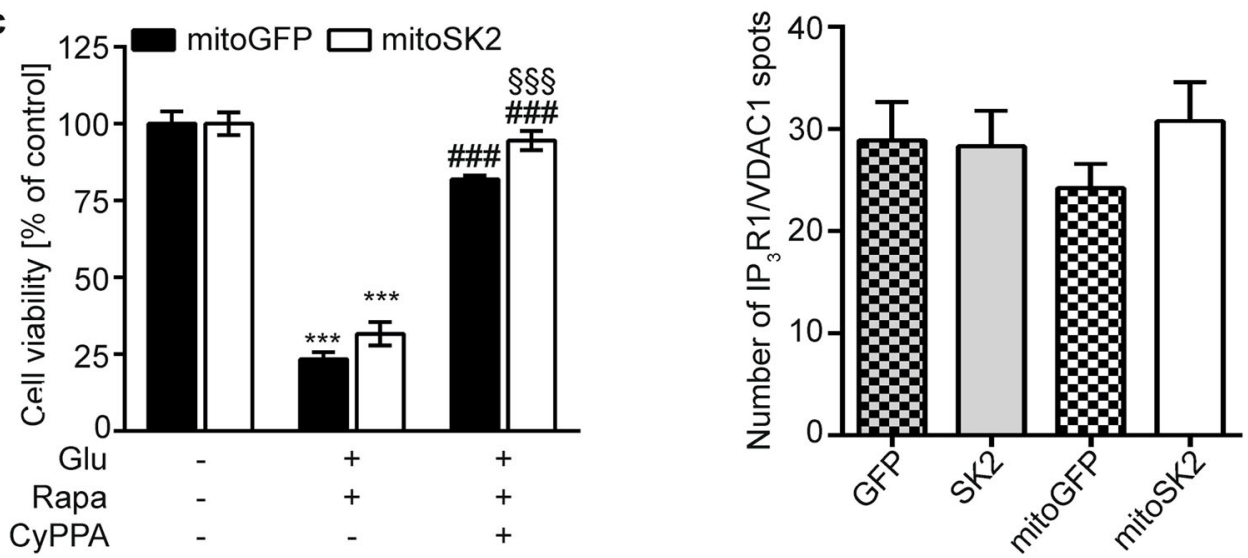

Fig. 4 Activation of SK channels protects against neuronal toxicity in conditions of enhanced EMC without affecting physical ER-mitochondrial coupling. a MTT Assay in HT22 cells transfected with ER-mitochondrial linkers following treatment with glutamate (4 mM, $16 \mathrm{~h}$ ) in the presence or absence of rapamycin $(100 \mathrm{nM})$ and/or CyPPA $\left(10 \mu \mathrm{M}\right.$, gray bars). Data are presented as mean $\pm S D, n=6,{ }^{* * *} p<0.0001$. b, $\mathbf{c}$ MTT assay in HT22 cells transfected with linker plasmids (EML) and (b) GFP or SK2 or (c) mitoGFP and mitoSK2 plasmids following treatment with glutamate $(4 \mathrm{mM}, 16 \mathrm{~h})$ in the presence or absence of rapamycin $(100 \mathrm{nM})$ and/or CyPPA $(10 \mu \mathrm{M})$. Data are presented as mean \pm SD, $n=$ $6-8$, Student's t-test, ${ }^{* *} p<0.01,{ }^{* * *} p<0.0001,{ }^{*}$ compared to control, ${ }^{*}$ compared to glutamate+rapamycin, ${ }^{\S}$ compared to GFP in the same treatment. In situ proximity ligation assay (PLA) in $\mathbf{d} \mathrm{HT} 22$ cells treated for $24 \mathrm{~h}$ with vehicle (DMSO) or $10 \mu \mathrm{M}$ CyPPA. In situ PLA in e HT22 cells transfected for $48 \mathrm{~h}$ with either GFP control plasmid or SK2 channel plasmid (left panels, light gray bars), or with mitoGFP control plasmid or mitoSK2 plasmid (right panels, white bars). Upper panels: representative images after in situ PLA, blue: DAPI, scale bar: $20 \mu \mathrm{M}$. Lower panels: quantification of IP ${ }_{3} \mathrm{R} 1 /$ VDAC1 spots on the analyzed pictures. Data are presented as mean \pm SEM, $n=25-30$ per condition 
bifunctional linkers to establish a stable platform for increased ER-mitochondrial interactions. Glutamateinduced cell death, starting approximately $12 \mathrm{~h}$ following induction, was more pronounced in cells transfected with the linkers compared to control cells. Thus, our findings indicate that EMC is a critical factor that potentiates neuronal damage generated by glutamate exposure.

SK channels, which are involved in controlling synaptic plasticity and neuronal firing, preserve cell survival in multiple disease paradigms by regulating $\mathrm{Ca}^{2+}$ signaling and mitochondrial function ${ }^{39,40}$. Despite their localization at the plasma membrane, recent studies have identified intracellularly expressed SK channels in the ER membrane and the $\mathrm{IMM}^{16,17,39,41}$ where they provide protection against ER stress and/or mitochondrial demise. Here, we show that activation of SK channels by the pharmacological SK2/3 channel activator CyPPA was able to protect against glutamate toxicity even in conditions of enhanced EMC that was associated with increased $\left[\mathrm{Ca}^{2+}\right]_{\mathrm{m}}$ uptake and impaired mitochondrial respiration. Interestingly, the overexpression of either wild-type or mitoSK2 channels enhanced the neuroprotective effects of CyPPA following glutamate treatment. Wild-type SK2 channels partially co-localized with the $\mathrm{ER}^{16}$ suggesting a contribution of SK channels from both, ER and mitochondria, to signaling along the MAM interface which might contribute to the observed protection during EMC enhancement. This concept is further supported by the fact that the physical association via $\mathrm{IP}_{3} \mathrm{R} 1-\mathrm{VDAC} 1$ interactions was not changed by SK channel activation and/or overexpression. These results highlight that SK channel activation is a promising approach to prevent neurodegeneration in conditions of glutamate toxicity even if neuronal cells are facing additional stress, such as increased $\left[\mathrm{Ca}^{2+}\right]_{\mathrm{m}}$ flux along the ER-mitochondrial axis and reduced mitochondrial respiration.

In conclusion, we show that strengthening EMC using genetically encoded linkers created a stable platform for ER-mitochondrial interactions, which enhanced $\left[\mathrm{Ca}^{2+}\right]_{\mathrm{m}}$ uptake and impaired mitochondrial bioenergetics, thereby potentiating neuronal cell death initiated by glutamate. SK channel activation by CyPPA, known to provide protection in different in vitro models of neurodegeneration, conferred protection against glutamate toxicity even when stress was pronounced due to an increase in EMC. These findings open a new platform to study SK channelmediated protection in conditions of oxidative stress and mitochondrial dysfunction that are related to changes in ER-mitochondrial signaling.

\section{Materials and methods Cell culture}

HT22 cells were cultured in Dulbecco's modified Eagle Medium (Sigma Aldrich, Munich, Germany) supplemented with $10 \%$ heat-inactivated fetal calf serum (PAA Cölbe, Germany), $100 \mathrm{U} / \mathrm{mL}$ penicillin, $100 \mu \mathrm{g} / \mathrm{mL}$ streptomycin, and $2 \mathrm{mM} \mathrm{L}$-glutamine (Invitrogen, Karlsruhe, Germany) at $37^{\circ} \mathrm{C}$ and $5 \% \mathrm{CO}_{2}$. Plasmid transfection was performed using the attractene transfection reagent according to the manufacturer's fast-forward protocol (Qiagen, Hilden, Germany). HT22 cells were transfected with $1.2 \mu \mathrm{g}$ plasmid DNA and grown in a 6-well plate for $48 \mathrm{~h}$ followed by re-seeding into the appropriate plate format for subsequent experiments. HEK293T cells were transfected with $400 \mathrm{ng}$ plasmid DNA and grown in a white-walled 96-well plate for $48 \mathrm{~h}$. HT22 cells were challenged with glutamate (Sigma Aldrich, Munich, Germany).

\section{Cell viability}

Cell viability was determined based on the metabolic activity using the MTT assay at a final concentration of $0.5 \mathrm{~g} / \mathrm{L}$ by incubation for $1 \mathrm{~h}$ at $37^{\circ} \mathrm{C}$, followed by removal of the MTT and at least $1 \mathrm{~h}$ incubation at $-20^{\circ} \mathrm{C}$. After dissolving the resulting formazan in DMSO, the absorbance of each well was determined with the Synergy H1 Multi-Mode reader (Biotek, LA, USA) at $570 \mathrm{nM}$ and at $630 \mathrm{nM}$. Alternatively, cell viability was monitored in real-time with cell impedance measurements, using the $x^{x C E L L i g e n c e ~}$ system $^{42}$ (ACEA Biosciences). Cell impedance was normalized to the time of treatment (normalized cell index), which is defined as the starting point ( $t=$ $0 \mathrm{~h}$ ) of the experiment.

\section{Visualization of ER-mitochondrial contacts in living cells}

To evaluate ER-mitochondrial contact formation upon transfection with TOM70-FKBP-mRFP and either ER-9xFRB-CFP (both linkers, depicted as EML) or ER-Flipper-GFP (depicted as FL) followed by the rapamycin-induced heterodimerization, widefield fluorescence microscopy (DeltaVision Elite) studies were performed. Transfected HT22 cells $\left(2 \times 10^{5}\right.$ cells/well $)$ were grown on $\varnothing 25 \mathrm{~mm}$ coverslips (Menzel-Gläser, Thermo Fisher, Landsmeer, The Netherlands). After image acquisition in untreated conditions, rapamycin $(100 \mathrm{nM})$ was applied, and images were taken again after $5 \mathrm{~min}$ and $10 \mathrm{~min}$ at $60 \times$ magnification, pixel size $6.5 \mu \mathrm{m} \times 6.5 \mu \mathrm{m}, 2560 \times 2160$ pixels, speed $400 \mathrm{fps}$ at $512 \times 512$ pixel. The software adopted for acquisition and integrated deconvolution was softWoRx (on Linux CentOS 6.3 platform). Image overlays were done using the ImageJ software.

\section{$\left[\mathrm{Ca}^{2+}\right]_{\mathrm{m}}$ measurements}

HEK293T cells were transfected with wild type and mutant mitochondrial GFP-aequorin ( $\mathrm{mtGA}^{\mathrm{wt}}, \mathrm{mtGA}^{\mathrm{mut}}$ ) for $48 \mathrm{~h}$ in a clear bottom, white-walled 96-well plate (Greiner Bioscience, Frickenhausen, Germany). Briefly, mtGA constructs were reconstituted with native 
coelenterazine (Biotium, VWR Technologies, Darmstadt, Germany) in medium for $2 \mathrm{~h}$. Cells were then washed with PBS followed by addition of $100 \mu \mathrm{L}$ internal buffer (140 $\mathrm{mM} \mathrm{KCl}, 1 \mathrm{mM} \mathrm{KH_{2 }} \mathrm{PO}_{4}, 1 \mathrm{mM} \mathrm{MgCl}, 10 \mathrm{mM}$ glucose, $0.1 \mathrm{mM}$ EGTA, $20 \mathrm{mM}$ HEPES, $8 \mathrm{mM}$ Na-succinate, $4 \mathrm{mM}$ Na-pyruvate, $4 \mathrm{mM}$ MgATP). $\mathrm{Ca}^{2+}$ uptake was initiated by addition of $50 \mathrm{mM} \mathrm{CaCl}_{2}$ or $500 \mu \mathrm{M}$ carbachol in internal buffer supplemented with $2.5 \mathrm{mM}$ MgEDTA following background measurement for $5 \mathrm{~s}$. Final sensor saturation was performed by addition of lysis buffer (140 $\mathrm{mM} \mathrm{KCl}, 10 \mathrm{mM} \mathrm{CaCl}_{2}, 1 \%$ TritonX100). Luminescence was recorded using the FluoStar OPTIMA plate reader (BMG Labtech, Offenbach, Germany). Each condition was measured at least in triplicate, and $\mathrm{Ca}^{2+}$ uptake was calculated as the total luminescent counts per second (L) from the time of stimulation $(t=5 \mathrm{~s})$ until the time of final cell lysis $(t=30 \mathrm{~s})$. Values were normalized to the initial value. For $\mathrm{mtGA}^{\mathrm{wt}}$ measurements, these values were additionally normalized to the total luminescence $(t=0 \mathrm{~s}$ until $t=45 \mathrm{~s})$. The sensor was calibrated with increasing $\mathrm{CaCl}_{2}$ concentrations (0, 10, 20, 30, 40, 50, 100 $\mathrm{mM}$ ) resulting in a dose-dependent increase in the luminescent signal as determined by cps and the normalized luminescence $\left(L_{t=5-30} / L_{\text {total }}\right)$.

\section{Seahorse XF analysis}

HT22 cells were transfected with mitochondrial linkers and either ER-Flipper control plasmid or the ER linker in Seahorse XF 96-well plates (Seahorse Biosystems, Agilent Technologies, Waldbronn, Germany). Prior to the measurement, the medium was removed and replaced by $180 \mu \mathrm{L}$ assay medium containing $4.5 \mathrm{~g} / \mathrm{L}$ glucose, $2 \mathrm{mM} \mathrm{L-}$ glutamine, $1 \mathrm{mM}$ pyruvate $(\mathrm{pH} 7.35)$ for $1 \mathrm{~h}$ at $37^{\circ} \mathrm{C}$. The OCR was analyzed using the Seahorse XF Biosystem. Three baseline measurements $(3 \mathrm{~min} \mathrm{mix}, 0 \mathrm{~min}$ delay, $3 \mathrm{~min}$ measure $=3 / 0 / 3$ ) were recorded followed by injection of rapamycin $(50-200 \mathrm{nM})$ or medium and measurement for $2 \mathrm{~h}$ (3/5/3). Following EMC, mitochondrial function was assessed by injection of $4 \mu \mathrm{M}$ oligomycin (3/0/3), $50 \mu \mathrm{M}$ DNP (3/0/3), and $150 \mathrm{nM}$ rotenone, $1 \mu \mathrm{M}$ antimycin $\mathrm{A}$, and $50 \mathrm{mM}$ 2-DG (3/0/3). After injection of each compound, the OCR was determined and values were normalized before injection into port A.

\section{Quantitative real-time PCR}

CHOP and GAPDH expression were analyzed using quantitative real-time PCR (qPCR). Therefore, total RNA was extracted from HT22 cells transfected with TOM70mRFP and either ER-Flipper GFP (FL) or ER-CFP (EML) and treated with $100 \mathrm{nM}$ and $200 \mathrm{nM}$ rapamycin for $6 \mathrm{~h}$, using Trizol RNA extraction (TRI Reagent Solution, Applied Biosystems, Landsmeer, Netherlands). The cDNA was synthesized from 500-1000 ng RNA using the
Reverse Transcriptase System (Promega, Madison, WI, USA), and the following protocol: $10 \mathrm{~min} 25^{\circ} \mathrm{C}, 45 \mathrm{~min}$ $42^{\circ} \mathrm{C}, 5 \mathrm{~min} 99^{\circ} \mathrm{C}$. The qPCR was performed with SYBR Green (Roche Diagnostics, Almere, Netherlands) and the following protocol including a final step to generate the melting curve: $2 \min 95^{\circ} \mathrm{C}, 10 \min 95^{\circ} \mathrm{C}, 45 \times\left(30 \mathrm{~s} 95^{\circ} \mathrm{C}\right.$, 30 s $60^{\circ} \mathrm{C}, 30$ s $72^{\circ} \mathrm{C}$ ), 30 s $95^{\circ} \mathrm{C}, 30$ s $55^{\circ} \mathrm{C}, 30$ s $95^{\circ} \mathrm{C}$. The qPCR was performed in an Eco Illumina (Illumina, Eindhoven, Netherlands). For analysis, the LinRegPCR software $^{43}$ was used to calculate NO values which were normalized to N0 of Gapdh as an internal control. Data are acquired in duplicates from three independent experiments.

The following murine primers were used: Chop (5' CAT ACACCACCACACCTGAAAG $3^{\prime}$ and 3' CCGTTTCCT AGTTCTTCCTTGC 5') and Gapdh (5' GGAGAGTG TTTCCTCGTCCC $3^{\prime}$ and $3^{\prime}$ ATGAAGGGGTCGTTG ATGGC $\left.5^{\prime}\right)$.

\section{In situ proximity ligation assay}

An optimized in situ proximity ligation assay (PLA) targeting the $\mathrm{IP}_{3} \mathrm{R} / \mathrm{VDAC} 1$ complex at the MAM interface was performed, as previously described ${ }^{44,45}$. Briefly, following CyPPA treatment or transfection with GFP/SK2 constructs, HT22 cells cultured on $35 \mathrm{~mm}$ glass bottom dishes (MatTek, Ashland, MA, USA) were fixed with 4\% PFA for $10 \mathrm{~min}$, permeabilized using $0.3 \% \mathrm{PBS} /$ TritonX100 for $30 \mathrm{~min}$, and saturated. The in situ PLA was performed according to the manufacturer's protocol: VDAC1 (mouse anti-VDAC1 primary antibody) and $\mathrm{IP}_{3} \mathrm{R} 1$ (rabbit anti- $\mathrm{IP}_{3} \mathrm{R} 1$ primary antibody) were probed followed by addition of the secondary antibodies anti-mouse and antirabbit IgG (PLA probe MINUS and PLUS) conjugated to complementary oligonucleotide extensions. If target proteins were within $40 \mathrm{nM}$ proximity, the oligonucleotides could hybridize with the connector oligonucleotides to form a circular DNA template, which was ligated and amplified to create a single-stranded DNA product. The DNA product is covalently attached to one of the proximity probes to allow detection of hybridized Texas redlabeled oligonucleotide probes. Each red fluorescent dot represents a site of $\mathrm{IP}_{3} \mathrm{R} 1-\mathrm{VDAC} 1$ interaction. Duolink II mounting medium containing DAPI 18 (Sigma Aldrich, Munich, Germany) was used for mounting, and preparations were analyzed with a Zeiss inversed fluorescent microscope at $63 \times$ magnification. Quantification of signals (number of red dots per cell) was done using the BlobFinder software. Experiments were performed in triplicate, $n=25-30$ pictures per conditions.

\section{Statistical analysis}

Statistical significance was assessed using the unpaired Student's $t$-test or Analysis of variance (ANOVA) and Scheffé's test for multiple comparisons, unless otherwise 
stated. $p$-values indicating statistically significant differences between the mean values are defined as follows: * $p$ $<0.05,{ }^{* * *} p<0.01$, and ${ }^{* * * *} p<0.001$.

\section{Acknowledgements}

We would like to thank Prof. Györgyi Hajnoczky for kindly providing the ER-mitochondrial linker plasmids, Prof. Ben N.G. Giepmans for providing the ER-Flipper-GFP plasmid, and Dr. Maria Alonso for providing mitochondrial GFPaequorin constructs. Furthermore, we would like to thank Prof. Dr. Moritz Bünemann and Prof. Dr. Cornelius Krasel for providing HEK293T cells, Prof. Frank J. Dekker and Dr. Nick Eleftheriadis for providing CyPPA, and Klaas Sjollema for his technical support on live-cell imaging. In addition, we acknowledge Lena Hoffmann for performing the osmolarity measurements. This work was supported by a grant from the Deutsche Forschungsgemeinschaft, DFG (DO 1525/3-1). A.M.D. is the recipient of a Rosalind Franklin Fellowship co-funded by the European Union and the University of Groningen.

\section{Author details}

${ }^{1}$ Institute of Pharmacology and Clinical Pharmacy, University of Marburg, 35043 Marburg, Germany. ${ }^{2}$ Faculty of Science and Engineering, Groningen Research Institute of Pharmacy (GRIP), Research School of Behavioural and Cognitive Neurosciences (BCN), Department of Molecular Pharmacology, University of Groningen, 9713 AV Groningen, The Netherlands. ${ }^{3}$ INSERM U1060, INRA U1235, Laboratoire CarMeN, Lyon University, Université Claude Bernard Lyon1, INSA-Lyon, F-69921 Oullins, France

\section{Conflict of interest}

The authors declare that they have no conflict of interest.

\section{Publisher's note}

Springer Nature remains neutral with regard to jurisdictional claims in published maps and institutional affiliations.

Supplementary Information accompanies this paper at (https://doi.org/ 10.1038/s41419-018-0590-1).

Received: 4 October 2017 Revised: 8 April 2018 Accepted: 12 April 2018 Published online: 22 May 2018

\section{References}

1. Tolar, M. et al. Truncated apolipoprotein E (ApoE) causes increased intracellular calcium and may mediate ApoE neurotoxicity. J. Neurosci. 19, 7100-7110 (1999).

2. Furukawa, K. et al. Plasma membrane ion permeability induced by mutant asynuclein contributes to the degeneration of neural cells. J. Neurochem. 97, 1071-1077 (2006).

3. Danzer, K. M. et al. Different species of a-synuclein oligomers induce calcium influx and seeding. J. Neurosci. 27, 9220-9232 (2007).

4. Federico, A. et al. Mitochondria, oxidative stress and neurodegeneration. J. Neurol. Sci. 322, 254-262 (2012).

5. Sutachan, J. J. et al. Cellular and molecular mechanisms of antioxidants in Parkinson's disease. Nutr. Neurosci. 15, 120-126 (2012).

6. Lemasters, J., Theruvath, T., Zhong, Z. \& Nieminen, A. Mitochondrial calcium and the permeability transition in cell death. Biochim. Biophys. Acta 1787, 1395-1401 (2010).

7. Chacon, E. \& Acosta, D. Mitochondrial regulation of superoxide by $\mathrm{Ca}^{2+}$ : an alternate mechanism for the cardiotoxicity of doxorubicin. Toxicol. Appl. Pharmacol. 107, 117-128 (1991).

8. Denton, R. M. \& McCormack, J. G. The role of calcium in the regulation of mitochondrial metabolism. Biochem. Soc. Trans. 8, 266-268 (1980).

9. Hansford, R. G. Physiological role of mitochondrial $\mathrm{Ca}^{2+}$ transport. J. Bioenergy Biomembr. 26, 495-508 (1994)

10. De Stefani, D. et al. VDAC1 selectively transfers apoptotic $\mathrm{Ca}^{2+}$ signals to mitochondria. Cell Death Differ. 19, 267-273 (2012).

11. Szabadkai, G. et al. Chaperone-mediated coupling of endoplasmic reticulum and mitochondrial $\mathrm{Ca}^{2+}$ channels. J. Cell Biol. 175, 901-911 (2006).
12. Rizzuto, R., Stefani, D., De, Raffaello, A. \& Mammucari, C. Mitochondria as sensors and regulators of calcium signalling. Nature 13, 566-578 (2012).

13. Hedskog, L. et al. Modulation of the endoplasmic reticulum-mitochondria interface in Alzheimer's disease and related models. Proc. Natl Acad. Sci. USA 110, 7916-7921 (2013).

14. Cali, T., Ottolini, D. \& Brini, M. Mitochondrial $\mathrm{Ca}^{2+}$ and neurodegeneration. Cell Calcium 52, 73-85 (2012).

15. Nishimura, A. L. et al. A mutation in the vesicle-trafficking protein VAPB causes late-onset spinal muscular atrophy and amyotrophic lateral sclerosis. Am. J. Hum. Genet. 75, 822-831 (2004).

16. Richter, M. et al. Activation of SK2 channels preserves ER-Ca ${ }^{2+}$ homeostasis and protects against ER stress-induced cell death. Cell Death Differ. 23, 1-14 (2015).

17. Honrath, B. et al. SK2 channels regulate mitochondrial respiration and mitochondrial $\mathrm{Ca}^{2+}$ uptake. Cell Death Differ. 1-13; https://doi.org/10.1038/ cdd.2017.2 (2017).

18. Giorgi, C., De Stefani, D., Bononi, A., Rizzuto, R. \& Pinton, P. Structural and functional link between the mitochondrial network and the endoplasmic reticulum. Int. J. Biochem. Cell Biol. 41, 1817-1827 (2009).

19. Csordás, G. et al. Imaging interorganelle contacts and local calcium dynamics at the ER-mitochondrial interface. Mol. Cell 39, 121-132 (2010).

20. Cárdenas, C. et al. Essential regulation of cell bioenergetics by constitutive InsP3 receptor $\mathrm{Ca}^{2+}$ transfer to mitochondria. Cell 142, 270-283 (2011).

21. Alonso, M. T., Navas-Navarro, P. \& García-Sancho, J. A Microplate-based bioluminescence assay of mitochondrial calcium uptake María. Mitochondria Pract. Protoc. 1567, 245-253 (2017).

22. Tan, S., Schubert, D. \& Maher, P. Oxytosis: a novel form of programmed cell death. Curr. Top. Med. Chem. 1, 497-506 (2001).

23. Cao, G. F. et al. Rapamycin sensitive mTOR activation mediates nerve growth factor (NGF) induced cell migration and pro-survival effects against hydrogen peroxide in retinal pigment epithelial cells. Biochem. Biophys. Res. Commun. 414, 499-505 (2011).

24. Herrik, K. F. et al. CyPPA, a positive SK3/SK2 modulator, reduces activity of dopaminergic neurons, inhibits dopamine release, and counteracts hyperdopaminergic behaviors induced by methylphenidate. Front. Pharmacol 3, 1-12 (2012).FEB.

25. Zündorf, G. \& Reiser, G. Calcium dysregulation and homeostasis of neural calcium in the molecular mechanisms of neurodegenerative diseases provide multiple targets for neuroprotection. Antioxid. Redox Signal. 14, 1275-1288 (2011).

26. Rizzuto, R. et al. Close contacts with the endoplasmic reticulum as determinants of mitochondrial $\mathrm{Ca}^{2+}$ responses. Science 280, 1763-1766 (1998).

27. Csordás, G. et al. Structural and functional features and significance of the physical linkage between ER and mitochondria. J. Cell Biol. 174, 915-921 (2006).

28. Paillusson, S. et al. There's something wrong with my MAM; the ERmitochondria axis and neurodegenerative diseases. Trends Neurosci. 39, 146-157 (2016).

29. Joshi, A. U., Kornfeld, O. S. \& Mochly-Rosen, D. The entangled ER-mitochondrial axis as a potential therapeutic strategy in neurodegeneration: a tangled duo unchained. Cell Calcium 60, 218-234 (2016).

30. Lebiedzinska, M., Szabadkai, G., Jones, A. W. E., Duszynski, J. \& Wieckowski, M. R. Interactions between the endoplasmic reticulum, mitochondria, plasma membrane and other subcellular organelles. Int. J. Biochem. Cell Biol. 41, 1805-1816 (2009).

31. Schwarzer, C., Barnikol-Watanabe, S., Thinnes, F. P. \& Hilschmann, N. Voltagedependent anion-selective channel (VDAC) interacts with the dynein light chain Tctex1 and the heat-shock protein PBP74. Int. J. Biochem. Cell Biol. 34, 1059-1070 (2002).

32. Egnatchik, R. A., Leamy, A. K, Jacobson, D. A., Shiota, M. \& Young, J. D. ER calcium release promotes mitochondrial dysfunction and hepatic cell lipotoxicity in response to palmitate overload. Mol. Metab. 3, 544-553 (2014).

33. Rapizzi, E. et al. Recombinant expression of the voltage-dependent anion channel enhances the transfer of $\mathrm{Ca}^{2+}$ microdomains to mitochondria. J. Cell Biol. 159, 613-624 (2002).

34. Bravo, R. et al. Increased ER-mitochondrial coupling promotes mitochondrial respiration and bioenergetics during early phases of ER stress. J. Cell Sci. 124, 2143-2152 (2011)

35. Cali, T., Ottolini, D., Negro, A. \& Brini, M. Enhanced parkin levels favor ERmitochondria crosstalk and guarantee $\mathrm{Ca}^{2+}$ transfer to sustain cell bioenergetics. Biochim. Biophys. Acta - Mol. Basis Dis. 1832, 495-508 (2013). 
36. Chen, Y. et al. Mitofusin 2-containing mitochondrial-reticular microdomains direct rapid cardiomyocyte bioenergetic responses via inter- organelle $\mathrm{Ca}^{2+}$ crosstalk. Circ. Res. 111, 863-875 (2013).

37. Deniaud, A. et al. Endoplasmic reticulum stress induces calcium-dependent permeability transition, mitochondrial outer membrane permeabilization and apoptosis. Oncogene 27, 285-299 (2008).

38. Breckenridge, D. G., Stojanovic, M., Marcellus, R. C. \& Shore, G. C. Caspase cleavage product of BAP31 induces mitochondrial fission through endoplasmic reticulum calcium signals, enhancing cytochrome $\mathrm{c}$ release to the cytosol. J. Cell Biol. 160, 1115-1127 (2003).

39. Dolga, A. et al. Subcellular expression and neuroprotective effects of SK channels in human dopaminergic neurons. Cell Death Dis. 5, e999 (2014).

40. Dolga, $\mathrm{A}$. et al. $\mathrm{K}_{\mathrm{Ca}} 2$ channels activation prevents $\left[\mathrm{Ca}^{2+}\right]_{i}$ deregulation and reduces neuronal death following glutamate toxicity and cerebral ischemia. Cell Death Dis. 2, e147 (2011).
41. Kuum, M. et al. Endoplasmic reticulum potassium-hydrogen exchanger and small conductance calcium-activated potassium channel activities are essential for ER calcium uptake in neurons and cardiomyocytes. J. Cell Sci. 125, 625-633 (2012).

42. Diemert, S. et al. Impedance measurement for real time detection of neuronal cell death. J. Neurosc M. 203, 69-77 (2012).

43. Ruijter, J. M. et al. Amplification efficiency: linking baseline and bias in the analysis of quantitative PCR data. Nucleic Acids Res. 37, e45 (2009).

44. Tubbs, E. \& Rieusset, J. Study of endoplasmic reticulum and mitochondria interactions by in situ proximity ligation assay in fixed cells. J. Vis. Exp. 1-5 https://doi.org/10.3791/54899 (2016).

45. Tubbs, E. et al. Mitochondria-associated endoplasmic reticulum membrane (MAM) integrity is required for insulin signaling and is implicated in hepatic insulin resistance. Diabetes 63, 3279-3294 (2014). 